\title{
DECREASING REARRANGEMENTS AND DOUBLY STOCHASTIC OPERATORS
}

BY

\author{
PETER W. DAY $\left({ }^{1}\right)$
}

\begin{abstract}
In this paper generalizations to measurable functions on a finite measure space $(X, \Lambda, \mu)$ of some characterizations of the Hardy-LittlewoodPblya preorder relation $\prec$ are considered. Let $\rho$ be a saturated, Fatou function norm such that $L^{\infty} C L^{\rho} C L^{1}$, and let $L^{\rho}$ be universally rearrangement invariant. The following equivalence is shown to hold for all $f \in L^{\rho}$ iff $(X, \Lambda, \mu)$ is nonatomic or discrete: $g<f$ iff $g$ is in the $\rho$-closed convex hull of the set of all rearrangements of $f$. Finally, it is shown that $g<f \in L^{1}$ iff $g$ is the image of $f$ by a doubly stochastic operator.
\end{abstract}

1. Introduction. In [5] and [6], G. H. Hardy, J. E. Littlewood, and G. Pólya introduced a preorder relation $\prec$ for $n$-tuples $x=\left(x_{1}, \ldots, x_{n}\right) \in \mathbf{R}^{n}$ of real numbers as follows. If $x \in \mathbf{R}^{n}$ let $x^{*}=\left(x_{1}^{*}, \ldots, x_{n}^{*}\right)$ denote the point obtained by rearranging the components of $x$ in decreasing order. Then for $x, y \in \mathbf{R}^{n}, y \prec x$ means

$$
\sum_{i=1}^{k} y_{i}^{*} \leq \sum_{i=1}^{k} x_{i}^{*}, \quad k=1, \cdots, n-1,
$$

with equality when $k=n$. Hardy, Littlewood, and Pólya characterized this preorder relation as follows [11].

(1.1) Theorem. The following are equivalent for $x, y \in \mathbf{R}^{n}$.

(1) $y \prec x$.

(2) $\sum_{i=1}^{n} \varphi\left(y_{i}\right) \leq \sum_{i=1}^{n} \varphi\left(x_{i}\right)$ for all continuous convex functions $\boldsymbol{\varphi}$ on $\mathbf{R}$.

(3) $y$ is in the convex bull of $\left\{z: z^{*}=x^{*}\right\}$.

(4) There is a doubly stochastic matrix $A$ sucb that $y=A x$.

Still another condition equivalent to $y \prec x$ has been given by Muirhead [12] (also see [16]).

Received by the editors April 30, 1971 and, in revised form, May 5, 1972.

AMS (MOS) subject classifications (1970). Primary 47B99; Secondary 28A65, 28A40, $46 \mathrm{E} 30$.

Key words and phrases. Doubly stochastic operator, decreasing rearrangement, measure preserving transformation, nonatomic measure, Riesz space, equimeasurability, finitely additive measure.

(1) Much of the paper is part of the author's doctoral thesis written under the direction of Professor W. A. J. Luxemburg at California Institute of Technology in 1970 while supported by an NSF Fellowship. The rest was obtained at Carnegie-Mellon University while supported by NSF Grant GU-2056.

Copyright $\bigcirc 1973$, American Mathematical Society 
It is of interest to try to generalize this theorem to functions in $L^{\rho}(X, \Lambda, \mu)$, where $\rho$ is a saturated Fatou norm such that $L^{\infty} \subset L^{\rho}, L^{\rho^{\prime}} \subset L^{1}$ and $L^{\rho}$ is universally rearrangement invariant (u.r.i.). The reader is referred to [9] for a discussion of these notions. A generalization to $L^{1}[0,1]$ of $(1) \Leftrightarrow(3) \Leftrightarrow$ (4) has been given by J. V. Ryff ([14], [15]). A generalization of $(1) \Leftrightarrow(2)$ has been given for $\sigma\left(L^{\infty}, L^{1}\right)$ by A. Grothendieck [2]. W. A. J. Luxemburg, [9] has independently given a generalization of $(1) \Leftrightarrow(2) \Leftrightarrow$ (3) for $\sigma\left(L^{\rho}, L^{\rho}\right)$.

After establishing some machinery in $\$ \$ 2$ and 3, we will in $\$ 4$ generalize (1) $\Leftrightarrow(4)$ for $L^{1}(X, \mu)$. Finally in $\$ 5$ we give a generalization of $(1) \Leftrightarrow$ (3) for the $\rho$-topology.

2. Preliminaries. Let $(X, \Lambda, \mu)$ be a finite measure space (m.s.), that is, $X$ is a nonempty point set, $\Lambda$ is a $\sigma$-algebra of subsets of $X, \mu$ is a nonnegative countably additive measure on $\Lambda$, and $a=\mu(X)<\infty$. We let $\int \cdot d \mu$ denote integration over $X$, and let $M(X, \mu)$ denote the extended real valued measurable functions on $X$. If $f \in M(X, \mu)$ its distribution function is defined by $d_{f}(s)=\mu(\{x: f(x)>s\})$ for all real $s$, and its decreasing rearrangement by $\delta_{f}(t)=\inf \left\{s: d_{f}(s) \leq t\right\}$ for $0 \leq t \leq \mu(X)$. The characteristic function of $E \in \Lambda$ is denoted by $1_{E}$, and the decreasing rearrangement of $1_{E}$ is denoted by $\delta_{E}$.

Let $\left(X_{1}, \Lambda_{1}, \mu_{1}\right)$ also be a finite m.s. with $\mu_{1}\left(X_{1}\right)=\mu(X)=a$. A map $\gamma$ : $X \rightarrow X_{1}$ is called measure preserving (m.p.) if $\mu\left(\gamma^{-1}[E]\right)=\mu_{1}(E)$ for all $E \in \Lambda_{1}$. If $f \in M(X, \mu)$ and $g \in M\left(X_{1}, \mu_{1}\right)$, then $f$ and $g$ are called equimeasurable (written $f \sim g)$ whenever $\delta_{f}=\delta_{g}$.

The Hardy-Littlewood-Pólya preorder relation is generalized as follows. If $f^{+} \in L^{1}(X, \mu)$ and $g^{+} \in L^{1}\left(X_{1}, \mu_{1}\right)$ then $g \prec \prec f$ (" $g$ is weakly majorized by $f$ ") means $\int_{0}^{t} \delta_{g} \leq \int_{0}^{t} \delta_{f}$ for all $0 \leq t \leq a$, and $g \prec f$ (" $g$ is majorized by $f$ ") means $g \prec \prec f$ and $\int_{0}^{a} \delta_{g}=\int_{0}^{a} \delta_{f}$.

Finally, $M$ denotes the set of all bounded, finitely additive real valued measures $\nu$ on $\Lambda$ such that $\nu(E)=0$ whenever $\mu(E)=0$. M is known to be a vector lattice, where if $\alpha, \beta \in \mathbb{M}$, then

$$
(\alpha \wedge \beta)(E)=\inf \left\{\alpha(T)+\beta\left(T^{c} \cap E\right): T \subseteq E, T \in \Lambda\right\}
$$

A measure $0 \leq \alpha \in \mathbb{M}$ is called purely finitely additive if the zero measure is the only countably additive measure between 0 and $\alpha$ in the lattice ordering. Every $\nu \in \mathbb{M}$ can be written $\nu=\nu_{c}+\nu_{p}$ where $\nu_{p}^{+}, \nu_{p}^{-}$are purely finitely additive, and $\nu_{c}$ is countably additive [17, Theorem 1.24]. Then $d \nu_{c}=g_{\nu} d \mu$ with $g_{\nu} \in L^{1}$.

3. Rounds of some functionals. Results of later sections depend on the following principle which is a corollary of the Hahn-Banach theorem for a locally convex topological vector space $V$ with continuous dual $V^{*}$. 
(3.1) Lemma. Let $K$ be a closed convex subset of $V$ and let $D \subset K$. Then $K$ is the closed convex bull of $D$ iff $\sup F[D] \geq \sup F[K]$ for all $F \in V^{*}$.

In this section results are given which pave the way for the use of this lemma.

A set $A \in \Lambda$ is called an atom of $(X, \Lambda, \mu)$ if $\mu(A)>0$, and for all $B \in \Lambda$ with $B \subset A$ we have either $\mu(B)=0$ or $\mu(A \backslash B)=0$. Any measurable function is essentially constant on every atom. A measure space is called nonatomic if it has no atoms. Although a nonatomic measure space is not measure-theoretically equivalent to $[0, \mu(X)]$ unless $(X, \Lambda, \mu)$ is separable, these two spaces can be related by a measure preserving map.

(3.2) Lemma. The following are equivalent.

(1) $(X, \Lambda, \mu)$ is nonatomic.

(2) There is a measure preserving map of $X$ into $[0, \mu(X)[$.

(3) If $v-u=\mu(X)$ then there is a m.p. map of $X$ into $[u, v[$.

(4) Every right continuous decreasing function on $[0, \mu(X)]$ is the decreasing rearrangement of a measurable function on $(X, \Lambda, \mu)$.

Proof. (1) $\Rightarrow(2)$. If $\phi$ is the function in [3, Lemma 7], then $\sigma(x)=\mu(X) \phi(x)$ is measure preserving. Alternatively, we may use $[4,41(2)]$ to define, for each $u=m / 2^{n}, n \geq 0,0 \leq m \leq 2^{n}$, sets $B_{u}$ such that $\mu\left(B_{u}\right)=u \mu(X)$ and $u<v$ implies $B_{u} \subset B_{v}$. Then $\{x: \sigma(x)>s\}=\bigcup\left\{B_{t}^{c}: t>s / a\right\}$ and we easily compute $\delta_{\sigma}(t)=\mu(X)-t$. (2) $\Rightarrow(3)$. If $\sigma: X \rightarrow[0, a[$ is m.p. and $v-u=a$, then $x \mapsto$ $\sigma(x)+u$ is a m.p. map of $X$ into $[u, v[$. (3) $\Rightarrow(4)$. Let $\sigma: X \rightarrow[0, a$ [ be m.p. If $F$ is decreasing and right-continuous on $[0, a]$ then $f=F \circ \sigma \sim F$, so $\delta_{f}=F$ by uniqueness of $\delta_{f} .(4) \Rightarrow(1)$. Let $f \in M(X, \mu)$ such that $\delta_{f}(t)=a-t$. Then $f$ is not constant on any subset of $X$ of positive measure so $X$ has no atoms.

Let $(X, \Lambda, \mu)$ be nonatomic and let $f \in M(X, \mu)$. If $A, B \in \Lambda$ have $\mu(A)=$ $\mu(B)$, then (3.2) may be used to define $f^{\prime}=$ a result of interchanging the values of $f$ on $A$ and $B$, as follows. Let $\sigma_{A}: A \rightarrow[0, \mu(A)]$ and $\sigma_{B}: B \rightarrow[0, \mu(B)]$ be m.p. Then $f^{\prime}=\delta_{f \mid A}{ }^{\circ} \sigma_{B}$ on $B,=\delta_{f \mid B}{ }^{\circ} \sigma_{A}$ on $A,=f$ elsewhere. Clearly $f^{\prime}$ $\sim f$.

Using (3.2) it is also easy to generalize to nonatomic m.s. a result of J. V. Ryff [15, Lemma 2] and G. Lorentz [7, p. 61] for [0, 1].

(3.3) Proposition (Lorentz-Ryff). If the finite m.s. $(X, \Lambda, \mu)$ is nonatomic and $f \in M(X, \mu)$ then there is a measure preserving map $\sigma: X \rightarrow[0, \mu(X)[$ such that $f=\delta_{f} \circ \sigma \mu$-a.e.

Proof. See [1, p. 26].

The next result is a generalization proved in $[9$, p. 102] of an inequality of Hardy and Littlewood. 
(3.4) Lemma. If $f, g \in M(X, \mu), a=\mu(X)<\infty$, and $\delta_{|f|} \delta_{|g|} \in L^{1}[0, a]$ then $f g \in L^{1}(X, \mu)$ and

$$
\int_{0}^{a} \delta_{f}(a-t) \delta_{g}(t) d t \leq \int f g d \mu \leq \int_{0}^{a} \delta_{f} \delta_{g}
$$

These inequalities bold also for all $0 \leq f, g \in M(X, \mu)$, even if $\delta_{f} \delta_{g} \notin$ $L^{1}[0, a]$.

It is a corollary of a theorem of Hardy [9, p. 94] that if $f^{\prime} \prec \prec f$ and both $\delta_{|f|} \delta_{|g|}$ and $\delta_{\left|f^{\prime}\right|} \delta_{|g|} \in L^{1}[0, a]$, then

$$
\int_{0}^{a} \delta_{\left|f^{\prime}\right|} \delta_{|g|} \leq \int_{0}^{a} \delta_{|f|} \delta_{|g|}
$$

If $f^{\prime} \prec f$ then in addition

$$
\int_{0}^{a} \delta_{f}(t) \delta_{g}(a-t) d t \leq \int f^{\prime} g d \mu \leq \int_{0}^{a} \delta_{f} \delta_{g} .
$$

If $f^{\prime} \prec f \in L^{1}(\mu)$ and $\delta_{|f|} \delta_{|g|} \in L^{1}[0, a]$ then, by approximating $|g|$ by non-

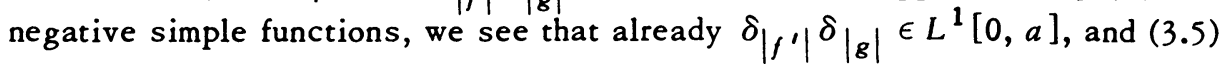
holds.

Because of its utility, Luxemburg has called a measure space adequate if $\max \left\{\int f g^{\prime} d \mu: g^{\prime} \sim g\right\}=\int_{0}^{a} \delta_{f} \delta_{g}$ for all $0 \leq f, g \in M(X, \mu)$, and he has asked for a characterization of such measure spaces $[9, \mathrm{p} .106]$. The following seems to be "adequate".

(3.6) Theorem. The following are equivalent for the finite m.s. $(X, \Lambda, \mu)$.

(1) $(X, \Lambda, \mu)$ is adequate.

(2) $(X, \Lambda, \mu)$ is nonatomic or consists only of atoms of equal measure.

(3) For all $A, B \in \Lambda$ we have

$$
\sup \left\{\int 1_{A} 1_{E} d \mu: 1_{E} \sim 1_{B}\right\}=\sup \{\mu(A \cap E): \mu(E)=\mu(B)\}=\int_{0}^{a} \delta_{A} \delta_{B} .
$$

Proof. $(2) \Rightarrow(1)$. Suppose $(X, \Lambda, \mu)$ is nonatomic. Let $\sigma: X \rightarrow[0, a]$ be m.p. such that $\delta_{f} \circ \sigma=f \mu$-a.e. Then $\int_{0}^{a} \delta_{f} \delta_{g}=\int\left(\delta_{f} \circ \sigma\right)\left(\delta_{g} \circ \sigma\right) d \mu=\int f g^{\prime} d \mu$, where $g^{\prime}=\delta_{g} \circ \sigma \sim g$. The proof when $(X, \mu)$ is discrete is similar [5, Theorem 368]. (1) $\Rightarrow(3)$ is obvious. It remains to prove $(3) \Rightarrow(2)$. Suppose (2) is not true. Then either $X$ has at least two atoms, $A, B$ with $0<\mu(B)<\mu(A)$; or $X$ has an atom $A$ and a nonatomic part $X_{0}$ of positive measure, in which case there is a $B \subset X_{0}$ such that $0<\mu(B)<\mu(A)$. In either case, for all $E \in \Lambda$ with $1_{E} \sim 1_{B}$ we have $\mu(E)=\mu(B)$ and hence $\mu(A \cap E) \leq \mu(E)=\mu(B)<\mu(A)$, so $\mu(A \cap E)=$ 0 , but $\int_{0}^{a} \delta_{A} \delta_{B}=\mu(B)>0$.

Finally, it is necessary to determine $\sup \left\{\int b d \nu: b \sim f\right\}$ when $f \in L^{\infty}, \nu \in \mathbb{M}$, and $(X, \Lambda, \mu)$ is nonatomic. 
(3.7) Lemma. Suppose $0<\alpha, \beta \in \mathbb{M}$ are purely finitely additive. If $\alpha \wedge \beta=$ 0 then there are sequences $\left\{A_{n}\right\}$ and $\left\{B_{n}\right\}$ such that
(a) $A_{n} \cap B_{n}=\varnothing$,
(c) $\beta\left(B_{n}\right) \uparrow \beta(X)$,
(b) $a\left(A_{n}\right) \uparrow a(X)$,
(d) $\mu\left(A_{n}\right)$ and $\mu\left(B_{n}\right) \rightarrow 0$.

The proof is straightforward using [17, 1.1.1 and Theorem 1.22].

(3.8) Lemma. Suppose $(X, \Lambda, \mu)$ is nonatomic, $A \cap B=\varnothing$ and $\mu(A), \mu(B)$ $\leq 1 / 4 \min \{\mu(S), \mu(T)\}$. Then there are sets $D \subset S$ and $E \subset T$ such that

(a) $\mu(D)=\mu(A), \mu(E)=\mu(B)$;

(b) $A, B, D$ and $E$ are pairwise disjoint.

Proof. $\mu(A)+\mu\left(A^{c} \cap B \cap S\right) / 2+\mu\left(A \cap B^{C} \cap S\right) / 2 \leq \mu(A)+\mu(B) / 2+\mu(A) / 2$ $\leq \mu(S) / 4+\mu(S) / 8+\mu(S) / 8=\mu(S) / 2$. Hence, $\mu(A) \leq \mu\left(A^{c} \cap B^{c} \cap S\right) / 2=$ $\left[\mu\left(A^{c} \cap B^{c} \cap S \cap T^{c}\right)+\mu\left(A^{c} \cap B^{c} \cap S \cap T\right)\right] / 2$. Similarly, $\mu(B) \leq$ $\left[\mu\left(A^{c} \cap B^{c} \cap S \cap T\right)+\mu\left(A^{c} \cap B^{c} \cap S^{c} \cap T\right)\right] / 2$. Since $(X, \Lambda, \mu)$ is nonatomic, $A^{c} \cap B^{c} \cap S \cap T=P \cup Q$ with $P \cap Q=\varnothing$ and $\mu(P)=\mu(Q)$. Hence $\mu(A) \leq$ $\mu\left(\left[A^{c} \cap B^{c} \cap S \cap T^{c}\right] \cup P\right)$ so there is a $D \subset\left(A^{c} \cap B^{c} \cap S \cap T^{c}\right) \cup P$ such that $\mu(D)=\mu(A)$. Similarly for $E \subset\left(A^{c} \cap B^{c} \cap S^{c} \cap T\right) \cup Q$.

(3.9) Proposition. Suppose $(X, \Lambda, \mu)$ is nonatomic, let $\nu \in \mathbb{M}$ and $f \in L^{\infty}$. Then

$$
\sup \left\{\int b d \nu: b \sim f\right\}=\int_{0}^{a} \delta_{f} \delta_{g_{\nu}}+\nu_{p}^{+}(X) \text { ess } \sup f-\nu_{p}^{-}(X) \text { ess inf } f .
$$

Proof. Let $r=$ ess sup $f, s=$ ess inf $f$, let $\sigma: X \rightarrow[0, a]$ be m.p. such that $\delta_{g_{\nu}} \circ \sigma=g_{\nu} \mu$-a.e., let $b=\delta_{f} \circ \sigma$, and for $i \geq 1$ let $S_{i}=\{|f-r|<1 / i\}$ and $T_{i}^{\nu}=\{|f-s|<1 / i\}$. Let $\left\{A_{n}\right\}$ and $\left\{B_{n}\right\}$ satisfy (a) $-(\mathrm{d})$ in (3.7) with $\alpha=\nu_{p}^{+}$ and $\beta=\nu_{p}^{-}$. Using (d) and passing to subsequences if necessary, we may assume $\mu\left(A_{i}\right), \mu\left(B_{i}\right) \leq 1 / 4 \min \left\{\mu\left(S_{i}\right), \mu\left(T_{i}\right)\right\}$. Hence by (3.8) there are sets $D_{i} \subset S_{i}$ and $E_{i} \subset T_{i}$ such that $\mu\left(D_{i}\right)=\mu\left(A_{i}\right), \mu\left(E_{i}\right)=\mu\left(B_{i}\right)$, and $A_{i}, B_{i}, D_{i}$, $E_{i}$ are pairwise disjoint.

For each $i \geq 1$ let $b_{i}$ be a result of first interchanging the values of $b$ on $D_{i}$ and $A_{i}$, and then of interchanging the values of the resulting function on $E_{i}$ and $B_{i}$. Then $b_{i} \sim b \sim f$, and $\int b_{i} d \nu=\int b_{i} g_{\nu} d \mu+\int b_{i} d \nu_{p}^{+}-\int b_{i} d \nu_{p}^{-}$. Now $\int b_{i} g_{\nu} d \mu \rightarrow \int b g_{\nu} d \mu$ as $i \rightarrow \infty$. Indeed, if $G_{i}=A_{i} \cup B_{i} \cup D_{i} \cup E_{i}$ then $b_{i}=$ $b$ on $X \backslash G_{i}$, so

$$
\int b_{i} g_{\nu} d \mu=\int_{X-G_{i}} b g_{\nu} d \mu+\int_{G_{i}} b_{i} g_{\nu} d \mu,\left|\int_{G_{i}} b_{i} g_{\nu} d \mu\right| \leq\|b\|_{\infty} \int_{G_{i}}\left|g_{\nu}\right| d \mu \rightarrow 0
$$

as $i \rightarrow \infty$. For the rest, 


$$
\begin{aligned}
\left|\int b_{i} d \nu_{p}^{+}-r \nu_{p}^{+}(X)\right| & \leq \int_{A_{i}}\left|b_{i}-r\right| d \nu_{p}^{+}+\int_{X-A_{i}}\left|b_{i}-r\right| d \nu_{p}^{+} \\
& \leq \frac{1}{i} \nu_{p}^{+}(X)+\|b-r\|_{\infty}\left[\nu_{p}^{+}(X)-\nu_{p}^{+}\left(A_{i}\right)\right] \rightarrow 0 .
\end{aligned}
$$

Similarly $\left|\int b_{i} d \nu_{p}^{-}-s \nu_{p}^{-}(X)\right| \rightarrow 0$. Since $\int b g_{\nu} d \mu=\int\left(\delta_{f} \circ \sigma\right)\left(\delta_{g_{\nu}} \circ \sigma\right) d \mu=$ $\int_{0}^{a} \delta_{f} \delta_{g_{\nu}}$, the proof is finished.

4. Doubly stochastic operators. If $T: L^{1}\left(\mu_{1}\right) \rightarrow L^{1}(\mu)$ is bounded and linear, let $T^{*}$ denote the adjoint of $T$, defined by $\int g T f d \mu=\int f T^{*} g d \mu_{1}$, for all $f \in L^{1}\left(\mu_{1}\right)$ and $g \in L^{\infty}(\mu)$. Then $T^{*}: L^{\infty}(\mu) \rightarrow L^{\infty}\left(\mu_{1}\right)$ and $T$ is weakly continuous [13, p. 38, Proposition 12] (or use nets and the defining equation).

By analogy with the definition for matrices, we define a doubly stochastic (d.s.) operator to be a bounded, linear operator $T: L^{1}\left(\mu_{1}\right) \rightarrow L^{1}(\mu)$ such that (1) $T \geq 0$; (2) $T 1_{X_{1}}=1_{X}$; and (3) $T^{*} 1_{X}=1_{X_{1}}$. It is easy to see that whenever two d.s. operators can be composed, the result is d.s.

(4.1) Theorem. Let $T$ be a linear map of the simple functions of $L^{1}\left(\mu_{1}\right)$ into $L^{1}(\mu)$. The following are equivalent:

(1) $T$ extends to a d.s. operator on $L^{1}\left(\mu_{1}\right)$.

(2) $0 \leq T 1_{E} \leq 1_{X}$ and $\int T 1_{E} d \mu=\mu_{1}(E)$ for all $E \in \Lambda_{1}$.

(3) There is a linear extension of $T$ to $L^{1}\left(\mu_{1}\right)$ such that $T f \prec f$ for all $f \in L^{1}\left(\mu_{1}\right)$.

In (1) and (3) the extension is necessarily unique.

Proof. (1) $\Rightarrow(2)$ is trivial. (2) $\Rightarrow(3)$ is proved as in [9, p. 130, (ii)]. For (3) $\Rightarrow(1)$, prove $T \geq 0$ as in [14, p. 1381], and prove $T 1_{X_{1}}=1_{X}$ as in $[9,(6.2$. iii)]. To show $T^{*} 1_{X}=1_{X}$ is easy. To see that the extensions are unique, note that $T \geq 0$ implies $(T f)^{+} \leq T f^{+}$and $(T f)^{-} \leq T f^{-}$, so $T$ is a contraction in both the $L^{1}$ and $L^{\infty}$ norms.

Remark. (i) (2) $\Leftrightarrow$ (3) was first proved by J. V. Ryff for $L^{1}[0,1]$.

(ii) $(1) \Rightarrow$ (3) here generalizes (1.1) (4) $\Rightarrow$ (1).

(4.2) Proposition. If $T: L^{1}\left(\mu_{1}\right) \rightarrow L^{1}(\mu)$ is d.s. then $T^{*}$ bas a unique extension to a d.s. map of $L^{1}(\mu) \rightarrow L^{1}\left(\mu_{1}\right)$.

Proof. We verify (4.1.2) for $T^{*}$. Let $E \in \Lambda$. For all $A \in \Lambda_{1}, \int_{A} T^{*} 1_{E} d \mu_{1}=$ $\int 1_{E} T 1_{A} d \mu$, and $0 \leq \int 1_{E} T 1_{A} d \mu \leq \int T 1_{A} d \mu=\mu_{1}(A)=\int{ }_{A} 1_{X_{1}} d \mu_{1}$, so $0 \leq$ $T^{*} 1_{E} \leq 1_{X_{1}}$. The rest is easy.

(4.3) Example. $T_{\gamma}$. If $\gamma: X \rightarrow X_{1}$ is m.p., define $T_{\gamma} f=f \circ \gamma$ for all $f \epsilon$ $L^{1}\left(\mu_{1}\right)$. Then $T_{\gamma} f \sim f$, so $T_{\gamma}$ is d.s. Of more importance, $T_{\gamma}^{*} T_{\gamma} f=f$ for all $f \in L^{1}\left(\mu_{1}\right)$. Indeed, for all $A \in \Lambda_{1}$ and $f \in L^{1}\left(\mu_{1}\right), \int 1_{A} T_{\gamma}^{*} T_{\gamma} f d \mu_{1}=$ $\int T_{\gamma} 1_{A} T_{\gamma} f d \mu=\int\left(1_{A} \circ \gamma\right)(f \circ \gamma) d \mu=\int 1_{A} f d \mu_{1}$ (see [15, Lemma 3]).

(4.4) Example. $T_{\mu}$ [9, p. 99]. The conditional expectation operator determined by a $\sigma$-subalgebra of $\Lambda$ is easily shown to be d.s. using (4.1). An important ex- 
ample is the d.s. operator $T_{\mu}$ which arises when a finite m.s. is embedded in a nonatomic m.s. Note that a finite or $\sigma$-finite m.s. has at most countably many atoms, so $X=X_{0} \cup \bigcup_{n \in P} A_{n}$, where $X_{0}$ is nonatomic, each $A_{n}$ is an atom, $P=\{1, \ldots, k\}$ or $P=\{1,2,3, \ldots\}$, and $\mu\left(A_{i} \cap A_{j}\right)=0$ whenever $i \neq j$.

We embed $(X, \Lambda, \mu)$ in a nonatomic m.s. $\left(X^{\#}, \Lambda^{\#}, \mu^{\#}\right)$ as follows. Let $I\left[a_{n}, b_{n}\right]$ be disjoint intervals of $\mathrm{R}$ with endpoints $a_{n}$ and $b_{n}$, such that $b_{n}-$ $a_{n}=\mu\left(A_{n}\right), n \in P$. Then define

$$
X^{\#}=X_{0} \cup \bigcup_{n \in P} I\left[a_{n}, b_{n}\right] ;
$$

$E \in \Lambda^{\#}$ iff $E=E_{0} \cup \bigcup_{n \in P} J_{n}$ where $E_{0} \in X_{0} \cap \Lambda$ and $J_{n} \subset I\left[a_{n}, b_{n}\right]$ is Lebesgue measurable; and $\mu(E)=\mu\left(E_{0}\right)+\Sigma_{n \in P} m\left(J_{n}\right)$ where $m=$ Lebesgue measure. Each $f \in M(X, \mu)$ is identified with $f^{\#}=f 1_{X_{0}}+\Sigma_{n \in P}\left(f \mid A_{n}\right) 1_{I}\left[a_{n}, b_{n}\right]$. Clearly $f^{\#} \sim f$, so $\delta_{f} \#=\delta_{f}$.

Finally, we define

$$
T_{\mu} f=f 1_{X_{0}}+\sum_{n \in P}\left(\frac{1}{b_{n}-a_{n}} \int_{a_{n}}^{b_{n}} f\right) 1^{A_{n}} \quad \text { for all } f \in M\left(X^{\sharp}, \mu^{\sharp}\right)
$$

for which this makes sense. Then:

(1) $T_{\mu}: L^{1}\left(\mu^{\#}\right) \rightarrow L^{1}(\mu)$ is d.s.;

(2) for all $f \in L^{1}\left(\mu^{\#}\right)$ and $g \in M(X, \mu)$ such that $f g^{\#} \in L^{1}\left(\mu^{\#}\right)$ we have $T_{\mu}\left(g^{\#} f\right)=g T_{\mu} f$, so $T_{\mu} g^{\#}=g$ and $\int g^{\#} f d \mu^{\#}=\int g T_{\mu} f d \mu$.

We now give a generalization of $(1.1)(1) \Rightarrow(4)$. Let $\mathscr{D}\left(X_{1}, X\right)=\{T \mid T$ : $L^{1}\left(\mu_{1}\right) \rightarrow L^{1}(\mu)$ is d.s. $\}, \mathscr{D}_{f}\left(X_{1}, X\right)=\left\{T f: T \in \mathscr{I}\left(X_{1}, X\right)\right\}$, and $\Omega_{f}(X, \mu)=$ $\{g \in M(X, \mu): g<f\}$ for $f \in L^{1}\left(\mu_{1}\right)$. Usually we will abbreviate these sets as $\mathfrak{D}, \mathfrak{D}_{f}, \Omega_{f}$, respectively.

(4.8) Lemma (Ryff). $\mathcal{D}\left(X_{1}, X\right)$ is convex and compact in the weak operator topology determined by the linear functionals $T \mapsto \int f T g d \mu, f \in L^{1}(\mu), g \in$ $L^{\infty}\left(\mu_{1}\right)$.

The proof given in [15, p. 97] generalizes easily.

(4.9) Theorem. Let $f \in L^{1}\left(X_{1}, \mu_{1}\right)$. If $g \in M(X, \mu)$ then $g \prec f$ iff there is a doubly stochastic operator $T: L^{1}\left(\mu_{1}\right) \rightarrow L^{1}(\mu)$ such that $g=T$.

Proof. Let $f \in L^{1}\left(\mu_{1}\right)$. Clearly $\mathscr{D}_{f} \subset \Omega_{f}$, so it suffices to show that $\Omega_{f} \subset$ $D_{f}$. Now $D_{f}$ is a convex, weakly closed subset of $L^{1}(\mu)$, because it is the image of the compact, convex set $\mathscr{I}\left(X, X_{1}\right)$ under the continuous, linear map $T \mapsto$ $T^{*} f$. Letting $K=\overline{\operatorname{cov}} \Omega_{f}\left(=\Omega_{f}\right.$, actually, but we do not need this $)$, it suffices to show $K=\overline{\operatorname{cov}} \mathfrak{I}_{f}=\mathfrak{D}_{f}$. We do this using Lemma (3.1). Let $g \in L^{\infty}(\mu)$. Then 
$\sup \left\{\int g h d \mu: b \in K\right\}=\sup \left\{\int g h d \mu: b \in \Omega_{f}\right\} \leq \int_{0}^{a} \delta_{f} \delta_{g}=\int f^{\prime} g^{\#} d \mu^{\#}=\int g T_{\mu} f^{\prime} d \mu$ for some $f^{\prime} \in M\left(X^{\#}, \mu^{\#}\right)$ such that $f^{\prime} \sim f$. Let $\sigma: X^{\#} \rightarrow[0, a]$ and $\gamma: X_{1}^{\#} \rightarrow$ $[0, \bar{a}]$ be measure preserving such that $T_{\sigma} \delta_{f}{ }^{\prime}=f^{\prime}$ and $T_{\gamma} \delta_{f}=f$. Since $f^{\prime} \sim f$, $\delta_{f}^{\prime}=\delta_{f}$, so $T_{\mu} f^{\prime}=T_{\mu} T_{\sigma} \delta_{f}^{\prime}=T_{\mu} T_{\sigma} \delta_{f}=T_{\mu} T_{\sigma} T_{\gamma}^{*} f \in \mathfrak{T}_{f}$, and the proof is finished.

(4.10) Corollary. If $f_{1}, f_{2} \in L^{1}\left(X_{1}, \mu_{1}\right)$ and $g \in M(X, \mu)$ and $g<f_{1}+f_{2}$ then there are $g_{1}, g_{2} \in L^{1}(X, \mu)$ such that $g=g_{1}+g_{2}$ and $g_{1} \prec f_{1}$ and $g_{2} \prec f_{2}$.

This generalizes $[8$, p. 51$]$.

5. $\Omega(f)$ is the closed convex hull of $\Delta(f)$. If $f \in L^{1}(X, \mu)$ let $\Delta(f)=\{b \epsilon$ $M: b \sim f\}$ and $\Omega(f)=\{b \in M: b \prec f\}$. One way to generalize (1.1) (1) $\Leftrightarrow$ (3) is to give conditions on a Banach function space $B$ between $L^{\infty}$ and $L^{1}$ such that for all $f \in B, \Omega(f)$ is the norm closed convex hull of $\Delta(f)$. That $\Omega(f)$ is convex when $f \in L^{1}$ follows as in [9, p. 135].

We will consider the class of Banach function spaces $L^{\rho}$ described in detail in [9], and [10]. Recall that a Riesz function norm is a mapping $\rho: M^{+}(X, \mu) \rightarrow$ $[0, \infty]$ which is zero only at functions which are zero $\mu$-a.e., which is positive homogeneous, satisfies the triangle inequality, and which is increasing: $0 \leq f \leq$ $g$ implies $\rho(/) \leq \rho(g)$. The norm $\rho$ is said to be Fatou if $0 \leq f_{n} \uparrow f$ pointwise implies $\rho\left(f_{n}\right) \uparrow \rho(f)$. We extend $\rho$ to $M(X, \mu)$ be defining $\rho(f)=\rho(|f|)$ and let $L^{\rho}(X, \mu)$ denote those $f$ for which $\rho(f)<\infty$. If $A \in \Lambda$ implies there is a $B \in \Lambda$ with $B \subset A, \mu(B)>0$, and $\rho\left(1_{B}\right)<\infty$, then $\rho$ is said to be saturated. Associated with $\rho$ are $\rho^{\prime}$ and $\rho^{\prime \prime}$ defined by $\rho^{\prime}(f)=\sup \left\{\int|f g| d \mu: \rho(g) \leq 1\right\}$ and $\rho^{\prime \prime}=$ $\left(\rho^{\prime}\right)^{\prime}$.

We assume for the remainder of this section that $\rho$ is a saturated Fatou function norm such that $L^{\infty} \subset L^{\rho} \subset L^{1}$. Then $L^{\rho}$ is complete [10, Note II, p. 149] and $L^{\infty} \subset L^{\rho^{\prime}} \subset L^{1}$. We also assume that $\Omega(f) \subset L^{\rho}$ whenever $f \in L^{\rho}$, which is equivalent to having $\delta_{|f|} \delta_{|g|} \in L^{1}[0, a]$ whenever $f \in L^{\rho}$ and $g \in L^{\rho^{\prime}}[9$, p. 116]. Such spaces $L^{\rho}$ are called (u.r.i.) by Luxemburg. It follows as in [9, pp. 135, 136] that $\Omega(f)$ is $\rho$-closed and $\rho$-bounded for all $f \in L^{\rho}$.

(5.1) Proposition. If $L^{\rho} \neq L^{\infty}$, then $\left(L^{\rho}\right)^{*}=L^{\rho^{\prime}}$.

Proof. Now $\left(L^{\rho}\right)^{*} \subset\left(L^{\infty}\right)^{*} \approx \Re$. Suppose $f \in L^{\rho} \backslash L^{\infty}$, and let $\nu \in\left(L^{\rho}\right)^{*}$. Since $\left|\nu_{p}\right| \wedge\left|\nu_{c}\right|=0\left[17\right.$, Theorem 1.16], we have $\left|\nu_{c}\right|+\left|\nu_{p}\right|=|\nu| \in\left(L^{\rho}\right)^{*}$ [10, Note VII, Theorem 22.3], so $\left|\nu_{p}\right| \in\left(L^{\rho}\right)^{*}$ [10, Note VII, Theorem 22.4]. Now $\left|\nu_{p}\right| \ll \mu$, so every atom of $\mu$ is an atom of $\left|\nu_{p}\right|$. Then $\left|\nu_{p}\right|$ is both countably and purely finitely additive on each atom of $\mu$, so $\left|\nu_{p}\right|=0$ on the atoms of $\mu$, if any. If $\mu\left(X_{0}\right)>0$, where $X_{0}$ is the nonatomic part of $X$, and $\left|\nu_{p}\right|\left(X_{0}\right) \neq 0$, 
then the proof of (3.9) shows that $\sup \left\{\left|\int b d\right| \nu_{p}|: b \sim| f \mid\right\}=+\infty$, which contradicts $\left|\nu_{p}\right| \in\left(L^{\rho}\right)^{*}$ and $\rho$-boundedness of $\Omega(f)$. Thus $\left|\nu_{p}\right|\left(X_{0}\right)=0$, so $d \nu=g_{\nu} d \mu$, and $g_{\nu} \in L^{\rho^{\prime}}$ by the converse of Hölder's inequality [10, Note V, Theorem 14.1].

(5.2) Theorem. $\Omega(f)$ is the $\rho$-closed convex bull of $\Delta(f)$ for all $f \in L^{\rho}$ iff $(X, \Lambda, \mu)$ is adequate.

Proof. If $f \in L^{\rho}$, then Lemma (3.1) says that $\Omega(f)$ is the closed convex hull of $\Delta(f)$ iff

$$
\sup F[\Delta(f)] \geq \sup F[\Omega(f)] \quad \text { for all } F \in\left(L^{\rho}\right)^{*} \text {. }
$$

If $(X, \Lambda, \mu)$ is not adequate, then Theorem (3.6) says there are $A, B \in \Lambda$ such that

$$
\begin{aligned}
\sup \left\{\int 1_{A} 1_{E} d \mu: 1_{E} \sim 1_{B}\right\}=0<\mu(B)=\int_{0}^{a} \delta_{A} \delta_{B} \\
=\sup \left\{\int 1_{A} T_{\mu} 1_{E} d \mu: 1_{E} \sim 1_{B}, E \in \Lambda^{\#}\right\} \leq \sup \left\{\int 1_{A} b d \mu: b<1_{B}\right\} .
\end{aligned}
$$

Since $\left(L^{\rho}\right)^{*} \supset L^{\infty},(*)$ fails for $f=1_{A}$ and $F(\cdot)=\int \cdot 1_{B} d \mu$.

Conversely, suppose $(X, \Lambda, \mu)$ is adequate and let $f \in L^{\rho}$. If $(X, \Lambda, \mu)$ is discrete, then Theorem (1.1) gives the result. Thus let $(X, \Lambda, \mu)$ be nonatomic. Suppose first that $L^{\rho} \neq L^{\infty}$, so $\left(L^{\rho}\right)^{*}=L^{\rho^{\prime}}$. Let $g \in L^{\rho^{\prime}}$, and let $\sigma: X \rightarrow$ $[0, a]$ be m.p. such that $\delta_{g} \circ \sigma=g \mu$-a.e. Since $\delta_{|f|} \delta_{|g|} \in L^{1}[0, a]$ we have

$$
\sup \left\{\int b g d \mu: b \in \Omega(f)\right\} \leq \int_{0}^{a} \delta_{f} \delta_{g}=\int\left(\delta_{f} \circ \sigma\right)_{g} d \mu,
$$

so $(*)$ holds. If $L^{\rho}=L^{\infty}$, and $\nu \in \mathbb{M}$, then

$$
\sup \left\{\int b d \nu: b \in \Omega(f)\right\} \leq \int_{0}^{a} \delta_{f} \delta_{g_{\nu}}+\nu_{p}^{+}(X) \text { ess sup } f-\nu_{p}^{-}(X) \text { ess inf } f,
$$

so Proposition (3.9) shows that $(*)$ holds.

6. Problem. The following problem, suggested by the previous results, seems to be open:

Let $(X, \Lambda, \mu)$ be nonatomic, let $f \in L^{\infty}$, and $\nu \in \mathbb{M}$. Characterize $\left\{\int b d \nu\right.$ : $b \sim f\}$ as a subset of R. For example, [9, Theorem (9.1)] and the proof of Proposition (3.9) show that this set is dense in

$$
\left[\int_{0}^{a} \delta_{f}(t) \delta_{g_{\nu}}(a-t), \int_{0}^{a} \delta_{f} \delta_{g_{\nu}}\right]+\left\{r \nu_{p}^{+}(X)-s \nu_{p}^{-}(X): r, s \in R_{f}\right\}
$$

where $R_{f}$ is the essential range of $f$.

\section{REFERENC.ES}

1. Peter W. Day, Rearrangements of measurable functions, Thesis, California Institute of Technology, Pasadena, Calif., 1970. 
2. A. Grothendieck, Rearrangements de fonctions et inegalités de convexité dains les algèbres de von Neumann d'une trace, Séminaire Bourbaki, 1955, 113-01-113-13.

3. Paul R. Halmos, The range of a vector measure, Bull. Amer. Math. Soc. 54 (1948), 416-421. MR 9, 574 .

4. - Measure theory, Van Nostrand Reinholdt, New York, 1950. MR 11, 504.

5. G. H. Hardy, J. E. Littlewood and G. Pólya, Inequalities, Cambridge Univ. Press, Cambridge, 1934.

6. - Some simple inequalities satisfied by convex functions, Messenger Math. 58 (1929), $145-152$.

7. G. G. Lorentz, Bernstein polynomials, Math. Expositions, no. 8, Univ. of Toronto Press, Toronto, 1953. MR 15, 217.

8. G. G. Lorentz and T. Shimogaki, Interpolation theorems for operators in function spaces, J. Functional Analysis 2 (1968), 31-51. MR 41 \#2424.

9. W. A. J. Luxemburg, Rearrangement invariant Banach function spaces, Queen's Papers in Pure and Appl. Math., no. 10, Queen's University, Kingston, Ont., 1967, pp. 83114.

10. W. A. J. Luxemburg and A. C. Zaanen, Notes on Banach function spaces. I-VII, Nederl. Akad. Wetensch. Proc. Ser. A 66 = Indag. Math. 25 (1963), 135-147, 148-153, $239-250,251-263,496-504,655-668,669-681$. MR 26 \#6723a, b; 27 \#1119a, b; 28 H 1481; \# 5324a.

11. L. Mirsky, Results and problems in the theory of doubly-stochastic matrices, Z. Wahrscheinlichkeitstheorie und Verw. Gebiete 1 (1962/63), 319-334. MR 27 \#3007.

12. R. F. Muirhead, Some methods applicable to identities and inequalities of symmetric algebraic functions of $n$ letters, Proc. Edinburgh Math. Soc. 21 (1903), $144-157$.

13. A. P. Robertson and W. J. Robertson, Topological vector spaces, Cambridge Tracts in Math. and Math. Phys., no. 53, Cambridge Univ. Press, New York, 1964. MR 28 \# 5318.

14. J. V. Ryff, On the representation of doubly stochastic operators, Pacific J. Math. 13 (1963), 1379-1386. MR 29 \#474.

15. - Orbits of $L^{1}$ functions under doubly stochastic transformations, Trans. Amer. Math. Soc. 117 (1965), 92 - 100. MR 35 \# 762.

16. - On Muirhead's theorem, Pacific J. Math. 21 (1967), 567-576. MR 35 $\# 3023$.

17. Kosaku Yosida and Edwin Hewitt, Finitely additive measures, Trans. Amer. Math. Soc. 72 (1952), 46-66. MR 13, 543.

DEPARTMENT OF MATHEMATICS, CARNEGIE-MELLON UNIVERSITY, PITTSBURGH, PENNSYLVANIA 15213

Current address: Computation Center, Uppergate House, Emory University, Atlanta, Georgia 30322 